\title{
A Double-blind, Randomized, Multicenter Clinical Trial Investigating the Efficacy and Safety of Esomeprazole Single Therapy Versus Mosapride and Esomeprazole Combined Therapy in Patients with Esophageal Reflux Disease
}

\author{
Ju Yup Lee, ${ }^{1}$ Sung Kook Kim, ${ }^{2 *}$ Kwang Bum Cho, ${ }^{1}$ Kyung Sik Park, ${ }^{1}$ Joong Goo Kwon, ${ }^{3}$ Jin Tae Jung, ${ }^{3}$ Eun Young Kim, ${ }^{3}$ \\ Byung Ik Jang, ${ }^{4}$ Si Hyung Lee, ${ }^{4}$ and Daegu-Gyeongbuk Gastrointestinal Study Group (DGSG) \\ ${ }^{1}$ Department of Internal Medicine, Keimyung University School of Medicine, Daegu, Korea; ${ }^{2}$ Department of Internal Medicine, Kyungpook \\ National University Hospital, Daegu, Korea; ${ }^{3}$ Department of Internal Medicine, Catholic University of Daegu School of Medicine, Daegu, Korea; \\ and ${ }^{4}$ Department of Internal Medicine, Yeungnam University College of Medicine, Daegu, Korea
}

\section{Background/Aims}

We aim to evaluate the efficacy and safety of combination therapy in erosive reflux disease (ERD) patients by comparing endoscopic healing rates according to the Los Angeles classification for esomeprazole alone, and esomeprazole plus mosapride.

\section{Methods}

A total of 116 ERD patients were randomized to receive esomeprazole $40 \mathrm{mg}$ once daily plus mosapride $5 \mathrm{mg} 3$ times daily ( $\mathrm{E}+\mathrm{M}$ group), or esomeprazole plus placebo (E only group) for 8 weeks. Patients recorded gastroesophageal reflux disease (GERD) symptom questionnaire at weeks 4 and 8 . The primary endpoint was the endoscopic healing rate of ERD after 8 weeks of treatment.

\section{Results}

Endoscopic healing rates according to the Los Angeles classification was 32 (66.7\%) in the $\mathrm{E}+\mathrm{M}$ group and 26 (60.5\%) in the $\mathrm{E}$ only group, but there was no statistically significant difference between the groups. Only at 4 weeks, the total GERD symptom score changes relative to the baseline significantly improved in the $\mathrm{E}+\mathrm{M}$ group than that of the $\mathrm{E}$ only group $(-13.4 \pm 14.7 \mathrm{vs}-8.0 \pm 12.3$, $P=0.041)$, and upper abdominal pain and belching score changes showed significantly improved in the $\mathrm{E}+\mathrm{M}$ group than that of the E only group $(P=0.018$ and $P=0.013$, respectively).

\section{Conclusions}

The combination of a proton pump inhibitor with mosapride shows a tendency for upper abdominal pain, belching, and total GERD symptoms scores to improve more rapidly. This suggests that combination therapy with esomeprazole and mosapride will be useful for rapid improvement of specific GERD symptoms, such as upper abdominal pain and belching in ERD patients.

(J Neurogastroenterol Motil 2017;23:218-228)

Key Words

Gastroesophageal reflux; Gastrointestinal motility; Proton pump inhibitors; Therapeutics

Received: June 24, 2016 Revised: December 6, 2016 Accepted: January 2, 2017

(.) This is an Open Access article distributed under the terms of the Creative Commons Attribution Non-Commercial License (http://creativecommons. org/licenses/by-nc/4.0) which permits unrestricted non-commercial use, distribution, and reproduction in any medium, provided the original work is properly cited.

*Correspondence: Sung Kook Kim, MD, PhD

Department of Internal Medicine, Kyungpook National University Hospital, 130 Dongdeok-ro, Jung-gu, Daegu 41944, Korea Tel: +82-53-420-5517, Fax: +82-53-426-8873, E-mail: knuhkim@gmail.com 


\section{Introduction}

Gastroesophageal reflux disease (GERD) is one of the most common diseases that causes symptoms like regurgitation or heartburn or tissue injury due to reflux of gastric contents into the esophagus. ${ }^{1,2}$ When erosions or ulcers are also observed in the esophagus during endoscopy, it is defined as erosive reflux disease (ERD). GERD impairs the patient's quality of life (QOL) ${ }^{3}$ and thus requires appropriate treatment; up to now, medication with protein pump inhibitors (PPIs) has been considered the best therapy. ${ }^{4,5}$ The clinical guidelines for GERD published in Korea in 2012, ${ }^{6}$ as well as diagnosis and treatment guidelines for GERD published in the United States in $2013,{ }^{7}$ indicate that PPIs are the most effective drugs for the treatment of ERD, with a high level of evidence and a strong level of recommendation. GERD is a complex multifactorial disease, which can be caused by excessive gastroesophageal reflux, reduced esophageal clearance of refluxed gastric contents, increased gastric acid, or increased sensitivity to refluxed contents in the esophageal mucosa. ${ }^{8}$ Among these possible etiologies, however, PPIs only affect acid production, with no effect on other mechanisms. For this reason, several drugs are prescribed in combination to increase the therapeutic effect, and the most common of these are prokinetic agents. ${ }^{9}$ According to the 2012 Korean clinical guidelines for GERD, prokinetics can help improve symptoms in GERD patients when prescribed together with PPIs, ${ }^{6}$ but the current level of evidence is low, and the results of different studies are inconsistent.

Mosapride is a typical prokinetic agent, which not only promotes esophageal and gastric motility as a 5 -hydroxytryptamine receptor $4\left(5-\mathrm{HT}_{4}\right)$ agonist, but also reduces visceral hypersensitivity. $^{10}$ According to previous studies, PPI + mosapride combination therapy significantly improves ERD symptoms compared to PPI alone, but there was no major difference in the endoscopic healing rate. ${ }^{11} \mathrm{~A}$ recent meta-analysis concluded that the combination therapy partially helps to improve QOL, but that it has no effect on alleviating symptoms or endoscopic healing. ${ }^{12}$ However, the number of studies included was small, ERD and non-erosive reflux disease (NERD) were both included, and there was heterogeneity in the PPI and prokinetics used; there was especially a lack of studies on the endoscopic healing rate and symptoms improvement following combination therapy in ERD patients. Therefore, this clinical trial aims to evaluate the efficacy and safety of combination therapy in ERD patients by comparing endoscopic healing rate according to the Los Angeles (LA) classification for esomeprazole alone and esomeprazole + mosapride.

\section{Materials and Methods}

\section{Study Design and Participants}

This study was a double-blind, randomized, multicenter, investigator-initiated clinical trial, conducted with 116 participants between November 2012 and June 2015. Four university hospitals participated in the trial—Kyungpook National University Hospital, Keimyung University Dongsan Medical Center, Daegu Catholic University Medical Center, and Yeungnam University Medical Center. The trial was conducted in accordance with Korean Good Clinical Practice and the Helsinki Declaration. The trial followed a protocol that previously approved by the Korean Ministry of Food and Drug Safety, and the Institutional Review Boards of the all participating organizations. All subjects participating in the trial gave their informed, written consent. All procedures and data from the trial were subject to careful monitoring.

Adult men and women, 20-70 years of age, who had complained of GERD symptoms (eg, heartburn and gastric acid reflux), or atypical symptoms (eg, chest pain, globus sensation, etc) for the last 3 months and at least 2 days in the week prior to the trial, and who had been diagnosed with grade A-D esophagitis (LA classification) in an upper gastrointestinal endoscopy examination in the last 5 weeks, were included in the study.

Exclusion criteria were as follows: patients with no GERD symptoms; patients whose symptoms were determined by the investigator to result from irritable bowel syndrome; patients with symptoms indicative of severe or malignant diseases, including unintended weight loss, hematemesis, hematochezia, or jaundice; patients with severe liver dysfunction or liver disease; patients with severe renal diseases, including chronic renal disease or renal dysfunction; patients with uncontrolled diabetes, cerebrovascular disease, or patients diagnosed within the last 3 months with a disease that requires surgery during the clinical trial period; patients diagnosed with Zollinger-Ellison syndrome, primary esophageal motility disorder, esophageal stricture, duodenal or gastric ulcer, malignant disease of the upper gastrointestinal tract, pancreatitis, disorders of absorption, severe cardiovascular disease, or lung disease within the last 3 months; patients who had taken PPIs within the last 28 days, had undergone endoscopy within the last 2 weeks, or had taken daily histamine $\mathrm{H} 2$ receptor antagonist, sucralfate, prokinetics, or antacids daily up to the initial visit; and finally, pregnant or breastfeeding women, as well as female patients who were not willing to use contraception for the duration of the clinical trial period. 


\section{Intervention}

Two weeks prior to medication, participants signed the consent form and underwent physical examination, pregnancy testing, vital sign measurements, blood tests, and urinalysis; the extent of reflux esophagitis was graded according to the LA classification ${ }^{13}$ via upper endoscopy as follows: Grade A: one or more mucosal breaks no longer than $5 \mathrm{~mm}$, none of which extends between the top of the mucosal folds; Grade B: one or more mucosal breaks longer than $5 \mathrm{~mm}$, none of which extend between the top of 2 mucosal folds; Grade C: mucosal breaks that extend between the top of 2 or more mucosal folds, but which involve less than $75 \%$ of the overall esophageal circumference; Grade D: mucosal breaks which involve at least $75 \%$ of the esophageal circumference.

Participants deemed suitable for the experiment during the screening period underwent a second inclusion/exclusion criteria check; the participants were then randomly allocated to a group, and prescribed with the trial medications. The experimental group (E+M group) took Esomezol capsules (Esomeprazole strontium tetrahydrate 40 mg; Hanmi Pharmaceutical Co, Ltd, Seoul, Korea) once daily and Mosazal tablets (Mosapride citrate $5 \mathrm{mg}$; Hanmi Pharmaceutical Co, Ltd) 3 times daily for 8 weeks; the control group (E only group) took Esomezol capsules and placebo tablets with identical dosing frequency and duration.

Four- and eight-week post-medication vital signs, GERD symptoms, and compliance were assessed; at 8 weeks post-medication, a follow-up upper endoscopy was also performed. Random allocation was achieved by block randomization, and the study used a double-blind design to minimize bias. Medication with drugs that could affect the clinical trial was banned during the trial period.

\section{Assessment of Gastroesophageal Reflux Disease Symptoms}

In order to establish the severity of GERD symptoms, a questionnaire was used to evaluate the extent of heartburn, acid regurgitation, upper abdominal pain, belching, dyspepsia, and nausea on a 5-point Likert scale (0-4): 0, no symptoms; 1 , feel symptoms but can easily endure and not long; 2 , feel inconvenience due to symptoms but no interference with normal activity; 3 , feel marked inconvenience due to symptoms and interference with normal activity; 4, severe symptoms that cannot endure and marked interference with normal activity, and the frequency of each symptom on a 6-point Likert scale (0-5), and then multiplying the scores for severity and frequency was calculated. ${ }^{11}$ The total symptom score was calculated as the sum of each of the 6 sub-scores.
All participants were asked whether GERD symptoms had been sufficiently improved by 4 and 8 weeks of treatment. After the participants had completed a questionnaire about GERD symptoms, the rate of improvement for the total score, and for individual sub-scores were calculated. Additionally, changes in the frequency of patients with reductions in GERD related upper abdominal pain and dyspepsia after 8 weeks of treatment were evaluated.

\section{Assessment of Adverse Events}

After drug administration, adverse events (diarrhea, nausea, headache, dizziness, itching, urticaria, etc) and severe adverse events were investigated for each treatment group, and the incidence of each of these events was compared between the groups. Changes in general blood test results, blood chemistry tests, and urinalysis were examined at 4 and 8 weeks after treatment and compared to the baseline. Similarly, changes in vital signs were also analyzed at 4 and 8 weeks and compared to the baseline.

\section{Study Endpoints}

The primary endpoint was evaluated in terms of the proportion of patients who improved by at least 1 grade according to the LA classification between the endoscopy result prior to medication and endoscopy result after 8 weeks of medication. The secondary endpoint was improvement in the total GERD score and individual symptom scores relative to the baseline at 4 and 8 weeks of medication. Additionally, changes in the frequency of patients with reductions in GERD related upper abdominal pain and dyspepsia after 8 weeks of treatment were evaluated.

\section{Statistical Methods}

The number of participants was selected by calculating the number required to compare the primary efficacy outcome (healing rate by the LA classification method) between the groups. Given the predicted healing rates for the $\mathrm{E}+\mathrm{M}$ group and the $\mathrm{E}$ only group of $97 \%$ and $77 \%$, respectively, $80 \%$ power, $5 \%$ type 1 error, and a $20 \%$ dropout rate, the total number of participants required was determined to be 110 .

Among the participants initially allocated to a group, those who completed the whole trial were defined as the per-protocol (PP) population; those who took the trial drug at least once and underwent efficacy tests at least once were defined as the intention-to-treat (ITT) population, and the efficacy analysis for ITT population was also included. Background analysis, including demographic characteristics, was performed on all clinical trial participants randomly allocated to a group, safety analysis was performed on the safety 
population, and efficacy analysis was performed on the PP and ITT populations.

For continuous variables, the number of subjects and the mean \pm standard deviation is given; for categorical variables, the frequency and percentage are presented. All continuous variables were tested for normality, and depending on the results, they were analyzed with either a two-sample or paired sample $t$ test for normal distributions, or with the Mann-Whitney $U$ test or Wilcoxon signed rank test for non-parametric distributions. Categorical variables were analyzed with the Pearson Chi-squared test or Fisher's exact test. All analyses were performed as two-tailed tests with a significance level of $5 \%$, meaning that results with a $P$-value of less than 0.05 were considered statistically significant. SPSS for Windows ver 21.0 (SPSS Inc, Chicago, IL, USA) was used for statistical analysis.

\section{Results}

\section{Characteristics of the Participants}

A total of 117 patients were subject to screening, and after one was excluded due to withdrawal of consent, the remaining 116 participants were randomly allocated into groups, with 60 in the $\mathrm{E}+\mathrm{M}$ group and 56 in the E only group. A further 3 participants were excluded for not taking the trial drug, while 4 were excluded because they did not undergo efficacy indicator assessment. The remaining 109 participants ( $\mathrm{E}+\mathrm{M}$ group, 56 participants; E only group, 53 participants) were included in the ITT population. Finally, 91 participants (E+M group, 48 participants; E only group, 43 participants) were included in the PP population; 18 participants were excluded for protocol violations, adverse events, withdrawal of consent, or other reasons (Fig. 1).

The average age for the $\mathrm{E}+\mathrm{M}$ group was $54.9 \pm 11.1$ years

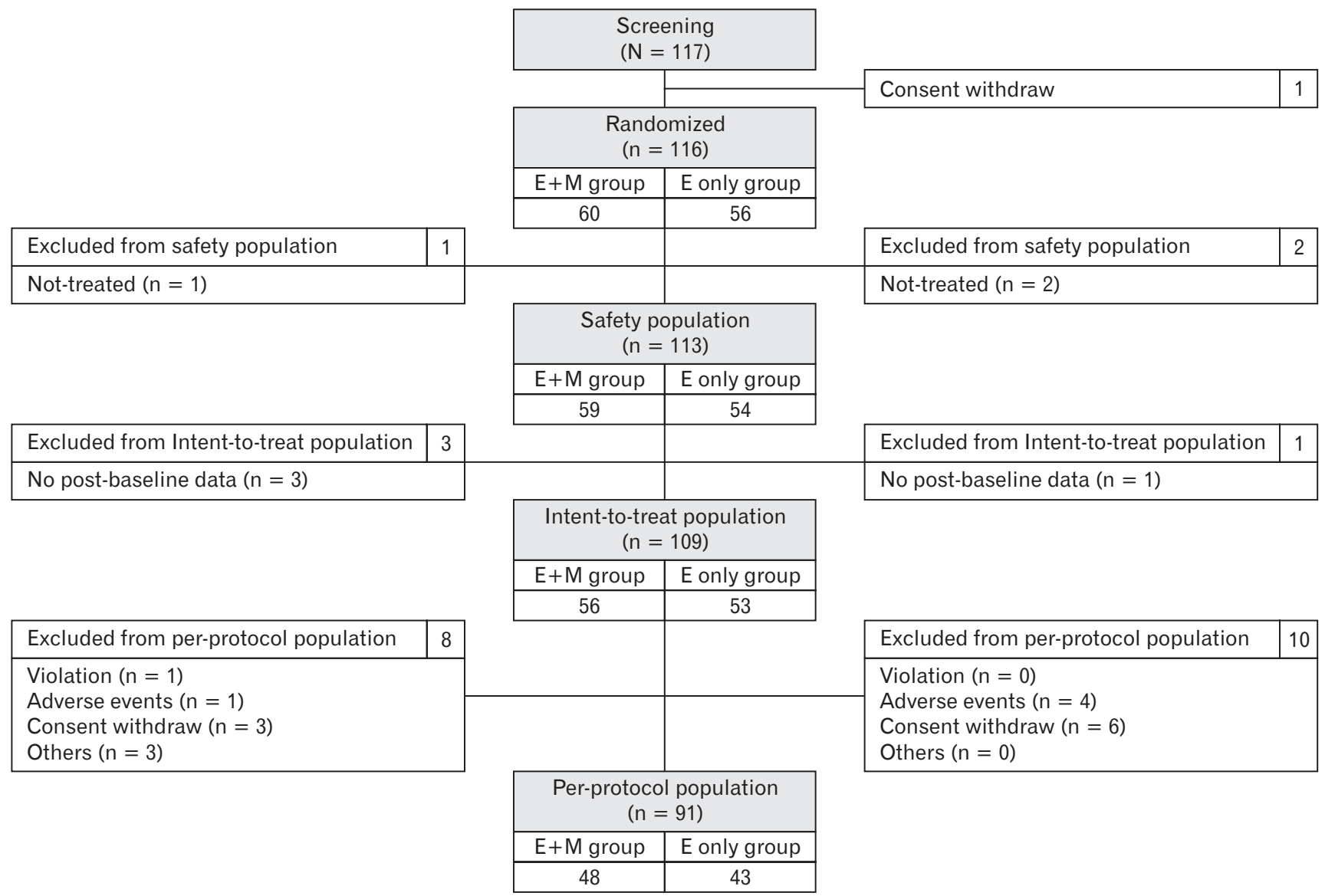

Figure 1. Subject disposition and analysis population of the study. E+M group, esomeprazole $40 \mathrm{mg}$ once daily plus mosapride $5 \mathrm{mg} 3$ times daily for 8 weeks; E only group, esomeprazole $40 \mathrm{mg}$ once daily plus placebo $5 \mathrm{mg} 3$ times daily for 8 weeks. 
and for the E only group was $55.8 \pm 9.9$ years. Both groups contained more men, with a male-to-female ratio of 38:22 (63.3\%) in the E+M group and 40:16 (71.4\%) in the E only group. There was also no significant difference between the groups in age, sex, smoking history, alcohol consumption, dietary and exercise habits, previous or concomitant medication history, disease history, or endoscopic LA classification (Table 1).

Table 1. Baseline Characteristics of the Study Subjects

\begin{tabular}{|c|c|c|c|c|}
\hline & $\begin{array}{c}E+M \text { group } \\
(n=60)\end{array}$ & $\begin{array}{c}\text { E only group } \\
(\mathrm{n}=56)\end{array}$ & Total & $P$-value \\
\hline Age, mean $\pm \mathrm{SD}$ & $54.9 \pm 11.1$ & $55.8 \pm 8.4$ & $55.3 \pm 9.9$ & 0.652 \\
\hline Height (cm) & $163.4 \pm 8.9$ & $166.4 \pm 8.6$ & $164.8 \pm 8.9$ & 0.064 \\
\hline Sex & & & & 0.465 \\
\hline Male & $38(63.3)$ & $40(71.4)$ & $78(67.2)$ & \\
\hline Female & $22(36.7)$ & $16(28.6)$ & $38(32.8)$ & \\
\hline Smoking status & & & & 0.661 \\
\hline Non-smoker & $32(53.3)$ & $32(57.1)$ & $64(55.2)$ & \\
\hline Ex-smoker & $16(26.7)$ & $11(19.6)$ & $27(23.3)$ & \\
\hline Smoker & $12(20.0)$ & $13(23.2)$ & $25(21.6)$ & \\
\hline Drinking status & & & & 0.565 \\
\hline Non-drinker & $20(33.3)$ & $22(39.3)$ & $42(36.2)$ & \\
\hline Ex-drinker & $5(8.3)$ & $8(14.3)$ & $13(11.2)$ & \\
\hline Mild drinker & $26(43.3)$ & $20(35.7)$ & $46(39.7)$ & \\
\hline Heavy drinker & $9(15.0)$ & $6(10.7)$ & $15(12.9)$ & \\
\hline Eating status & & & & 0.553 \\
\hline Regular, regimen & $30(50.0)$ & $26(46.4)$ & $56(48.3)$ & \\
\hline Regular, non-regimen & $19(31.7)$ & $15(26.8)$ & $34(29.3)$ & \\
\hline Irregular, regimen & $5(8.3)$ & $4(7.1)$ & $9(7.8)$ & \\
\hline Irregular, non-regimen & $6(10.0)$ & $11(19.6)$ & $17(14.7)$ & \\
\hline Exercise status & & & & 0.264 \\
\hline Non-exerciser & $15(25.0)$ & $17(30.4)$ & $32(27.6)$ & \\
\hline Irregular & $27(45.0)$ & $17(30.4)$ & $44(37.9)$ & \\
\hline Regular & $18(30.0)$ & $22(39.3)$ & $40(34.5)$ & \\
\hline Previous medications & & & & 0.964 \\
\hline No & $33(55.0)$ & $32(57.1)$ & $65(56.0)$ & \\
\hline Yes & $27(45.0)$ & $24(42.9)$ & $51(44.0)$ & \\
\hline Concomitant medication & & & & 1.000 \\
\hline No & $33(55.0)$ & $31(55.4)$ & $64(55.2)$ & \\
\hline Yes & $27(45.0)$ & $25(44.6)$ & $52(44.8)$ & \\
\hline Medical history & & & & 1 \\
\hline No & $1(1.7)$ & $0(0.0)$ & $1(0.9)$ & \\
\hline Yes & $59(98.3)$ & $56(100.0)$ & $116(100.0)$ & \\
\hline LA classificationed $^{\mathrm{a}}$ & & & & 0.749 \\
\hline Class A & $43(71.7)$ & $40(72.7)$ & $83(72.2)$ & \\
\hline Class B & $16(26.7)$ & $13(23.6)$ & $29(25.2)$ & \\
\hline Class C & $1(1.7)$ & $2(3.6)$ & $3(2.6)$ & \\
\hline Class D & $0(0.0)$ & $0(0.0)$ & $0(0.0)$ & \\
\hline
\end{tabular}

${ }^{a}$ Exclude 1 participant who did not match Los Angeles (LA) classification definition.

$\mathrm{E}+\mathrm{M}$ group, esomeprazole $40 \mathrm{mg}$ once daily plus mosapride $5 \mathrm{mg} 3$ times daily for 8 weeks; E only group, esomeprazole $40 \mathrm{mg}$ once daily plus placebo $5 \mathrm{mg} 3$ times daily for 8 weeks. 


\section{Efficacy Assessment}

\section{Endoscopic healing rates according to the Los Angeles classification}

Of the participants in the $\mathrm{PP}$ population, the number showing improved endoscopic findings according to the LA classification was $32(66.7 \%)$ in the E+M group and $26(60.5 \%)$ in the E only group ( $P=0.692$ ) (Table 2$)$. When the ITT population was analyzed, the number of participants showing improved endoscopic findings was $33(66.0 \%)$ in the $\mathrm{E}+\mathrm{M}$ group and $26(60.5 \%)$ in the E only group $(P=0.736)$ (Table 2$)$.

Table 2. Endoscopic Healing Rates According to the Los Angeles Classification

\begin{tabular}{|c|c|c|c|c|}
\hline \multirow{2}{*}{ Subjects } & \multirow{2}{*}{ Change } & $\mathrm{E}+\mathrm{M}$ group & E only group & \multirow{2}{*}{$P$-value } \\
\hline & & \multicolumn{2}{|c|}{ Frequency $(\%)$} & \\
\hline \multirow{2}{*}{$\begin{array}{l}\mathrm{PP} \\
\quad(\mathrm{n}=91)\end{array}$} & Cured & $32(66.7)$ & $26(60.5)$ & \multirow{2}{*}{0.692} \\
\hline & Not cured & $16(33.3)$ & $17(39.5)$ & \\
\hline \multirow{2}{*}{$\begin{array}{l}\text { ITT } \\
\qquad(\mathrm{n}=93)\end{array}$} & Cured & $33(66.0)$ & $26(60.5)$ & \multirow{2}{*}{0.736} \\
\hline & Not cured & $17(34.0)$ & $17(39.5)$ & \\
\hline
\end{tabular}

PP, per-protocol; ITT, intention-to-treat; E+M group, esomeprazole $40 \mathrm{mg}$ once daily plus mosapride $5 \mathrm{mg} 3$ times daily for 8 weeks; E only group, esomeprazole $40 \mathrm{mg}$ once daily plus placebo $5 \mathrm{mg} 3$ times daily for 8 weeks.

Table 3. Changes in Total and Individual Gastroesophageal Reflux Disease Symptom Scores After 4 and 8 Weeks of Medication (Per-protocol Analysis)

\begin{tabular}{|c|c|c|c|c|c|c|c|}
\hline & Baseline & Week 4 & Week 8 & $\begin{array}{l}\text { Changes from baseline } \\
\text { (Week } 4 \text { - baseline) }\end{array}$ & $P$-value & $\begin{array}{c}\text { Changes from baseline } \\
\text { (Week } 8 \text { - baseline) }\end{array}$ & $P$-value \\
\hline \multicolumn{8}{|l|}{ Total score } \\
\hline$E+M(n=48)$ & $18.94 \pm 18.08$ & $5.19 \pm 5.93^{\mathrm{a}}$ & $5.08 \pm 5.40^{\mathrm{a}}$ & $-13.75 \pm 15.15$ & \multirow{2}{*}{0.158} & $-13.85 \pm 16.37$ & \multirow{2}{*}{0.512} \\
\hline E only $(n=43)$ & $17.47 \pm 15.54$ & $7.91 \pm 8.93^{\mathrm{a}}$ & $5.60 \pm 8.40^{\mathrm{a}}$ & $-9.56 \pm 12.62$ & & $-11.86 \pm 11.90$ & \\
\hline \multicolumn{8}{|l|}{ Heartburn } \\
\hline $\mathrm{E}+\mathrm{M}(\mathrm{n}=48)$ & $4.40 \pm 4.29$ & $0.54 \pm 0.90^{\mathrm{a}}$ & $0.92 \pm 1.72^{\mathrm{a}}$ & $-3.85 \pm 4.27$ & \multirow{2}{*}{0.155} & $-3.48 \pm 4.28$ & \multirow{2}{*}{0.576} \\
\hline E only $(n=43)$ & $3.63 \pm 4.27$ & $1.05 \pm 1.62^{\mathrm{a}}$ & $0.65 \pm 1.48^{\mathrm{a}}$ & $-2.58 \pm 4.17$ & & $-2.98 \pm 4.25$ & \\
\hline \multicolumn{8}{|l|}{ Acid regurgitation } \\
\hline$E+M(n=48)$ & $3.96 \pm 4.97$ & $0.75 \pm 1.34^{\mathrm{a}}$ & $0.50 \pm 1.25^{\mathrm{a}}$ & $-3.21 \pm 4.69$ & \multirow{2}{*}{0.211} & $-3.46 \pm 4.79$ & \multirow{2}{*}{0.147} \\
\hline E only $(n=43)$ & $2.84 \pm 3.49$ & $0.74 \pm 1.24^{\mathrm{a}}$ & $0.67 \pm 1.57^{\mathrm{a}}$ & $-2.09 \pm 3.62$ & & $-2.16 \pm 3.47$ & \\
\hline \multicolumn{8}{|c|}{ Upper abdominal pain } \\
\hline$E+M(n=48)$ & $2.88 \pm 3.97$ & $0.65 \pm 1.04^{\mathrm{a}}$ & $0.79 \pm 1.76^{\mathrm{a}}$ & $-2.23 \pm 4.09$ & \multirow{2}{*}{0.156} & $-2.08 \pm 4.18$ & \multirow{2}{*}{0.703} \\
\hline E only $(\mathrm{n}=43)$ & $2.26 \pm 3.61$ & $1.09 \pm 2.01^{\mathrm{a}}$ & $0.47 \pm 1.56^{\mathrm{a}}$ & $-1.16 \pm 2.82$ & & $-1.79 \pm 2.95$ & \\
\hline \multicolumn{8}{|l|}{ Belching } \\
\hline $\mathrm{E}+\mathrm{M}(\mathrm{n}=48)$ & $3.81 \pm 4.53$ & $1.58 \pm 2.69^{\mathrm{a}}$ & $1.65 \pm 2.51^{\mathrm{a}}$ & $-2.23 \pm 3.16$ & \multirow{2}{*}{0.073} & $-2.17 \pm 3.96$ & \multirow{2}{*}{0.580} \\
\hline E only $(n=43)$ & $3.65 \pm 4.58$ & $2.72 \pm 3.47$ & $1.91 \pm 3.04^{\mathrm{a}}$ & $-0.93 \pm 3.67$ & & $-1.74 \pm 3.20$ & \\
\hline \multicolumn{8}{|l|}{ Dyspepsia } \\
\hline$E+M(n=48)$ & $2.81 \pm 4.66$ & $1.21 \pm 2.81^{\mathrm{a}}$ & $1.00 \pm 2.07^{\mathrm{a}}$ & $-1.60 \pm 4.00$ & \multirow{2}{*}{0.648} & $-1.81 \pm 3.73$ & \multirow{2}{*}{0.507} \\
\hline E only $(n=43)$ & $3.81 \pm 4.95$ & $1.81 \pm 2.97^{\mathrm{a}}$ & $1.42 \pm 3.12^{\mathrm{a}}$ & $-2.00 \pm 4.24$ & & $-2.40 \pm 4.60$ & \\
\hline \multicolumn{8}{|l|}{ Nausea } \\
\hline$E+M(n=48)$ & $1.08 \pm 2.02$ & $0.46 \pm 1.80$ & $0.23 \pm 0.78^{\mathrm{a}}$ & $-0.63 \pm 2.56$ & \multirow{2}{*}{0.739} & $-0.85 \pm 2.18$ & \multirow{2}{*}{0.884} \\
\hline E only $(n=43)$ & $1.28 \pm 2.32$ & $0.49 \pm 1.26^{\mathrm{a}}$ & $0.49 \pm 1.16^{\mathrm{a}}$ & $-0.79 \pm 2.12$ & & $-0.79 \pm 1.93$ & \\
\hline
\end{tabular}

${ }^{a}$ Compared within group (baseline and week 4 or 8 ): $P$-value by paired $t$ test, $P<0.05$ compared to baseline.

E+M group, esomeprazole $40 \mathrm{mg}$ once daily plus mosapride $5 \mathrm{mg} 3$ times daily for 8 weeks; E only group, esomeprazole $40 \mathrm{mg}$ once daily plus placebo 5 mg 3 times daily for 8 weeks. 
Changes in total and individual gastroesophageal reflux disease symptom scores after 4 and 8 weeks of medication

Changes in gastroesophageal reflux disease (total score) symptoms after 4 and 8 weeks of medication. In the PP analysis population, the total scores for GERD symptoms after 4 weeks of medication showed no statistically significant difference between the 2 treatment groups ( $P=0.095$ ). Both groups showed a significant decrease in total scores relative to baseline at 4 and 8 weeks after medication ( 4 and 8 weeks: $\mathrm{E}+\mathrm{M}$ group, $P<$ 0.001; E only group, $P<0.001$ ) (Table 3 ). In the ITT analysis population, the 2 treatment groups did show a significant difference in the absolute change in GERD total symptom scores relative to the baseline at 4 weeks $(P=0.041)$ (Table 4).

Changes in heartburn symptom after 4 and 8 weeks of medication. When the change in heartburn relative to the baseline at 4 and 8 weeks was analyzed for the 2 groups, a significant decrease was observed in both groups $(\mathrm{E}+\mathrm{M}$ group, $P<$
0.001; E only group, $P<0.001$; both at 4 and 8 weeks) (Table 3 ).

Changes in acid regurgitation symptom after 4 and 8 weeks of medication. The change in acid regurgitation scores relative to the baseline for the $\mathrm{PP}$ analysis population at 4 and 8 weeks after medication decreased significantly for both groups (E $+\mathrm{M}$ group, $P<0.001$; E only group, $P<0.001$; both at 4 and 8 weeks) (Table 3 ).

Changes in upper abdominal pain symptom after 4 and 8 weeks of medication. In the PP analysis population, changes in upper abdominal pain scores relative to the baseline at 4 and 8 weeks after medication, showed a statistically significant decrease in both groups (Table 3). In the ITT analysis population, there was a statistically significant difference between the 2 groups in upper abdominal pain score after 4 weeks $(P=0.030)$, and the improvement relative to the baseline after 4 weeks of medication was significantly higher in the $\mathrm{E}+\mathrm{M}$ group than in the $\mathrm{E}$ only group $(P$ $=0.018)$. The change at 4 weeks was analyzed relative to the base-

Table 4. Changes in Total and Individual Gastroesophageal Reflux Disease Symptom Scores After 4 and 8 Weeks of Medication (Intention-to-treat Analysis)

\begin{tabular}{|c|c|c|c|c|c|c|c|}
\hline & Baseline & Week 4 & Week 8 & $\begin{array}{l}\text { Changes from baseline } \\
\text { (Week } 4 \text { - baseline) }\end{array}$ & $P$-value & $\begin{array}{l}\text { Changes from baseline } \\
\text { (Week } 8 \text { - baseline) }\end{array}$ & $P$-value \\
\hline \multicolumn{8}{|l|}{ Total score } \\
\hline $\mathrm{E}+\mathrm{M}(\mathrm{n}=56)$ & $19.57 \pm 18.05$ & $6.16 \pm 6.96^{\mathrm{a}}$ & $6.13 \pm 6.59^{\mathrm{a}}$ & $-13.41 \pm 14.72$ & \multirow{2}{*}{0.041} & $-13.45 \pm 15.82$ & \multirow{2}{*}{0.234} \\
\hline E only $(\mathrm{n}=53)$ & $17.51 \pm 15.48$ & $9.49 \pm 10.67^{\mathrm{a}}$ & $7.30 \pm 10.46^{\mathrm{a}}$ & $-8.02 \pm 12.34$ & & $-10.21 \pm 12.05$ & \\
\hline \multicolumn{8}{|l|}{ Heartburn } \\
\hline $\mathrm{E}+\mathrm{M}(\mathrm{n}=56)$ & $4.27 \pm 4.19$ & $0.71 \pm 1.02^{\mathrm{a}}$ & $1.05 \pm 1.71^{\mathrm{a}}$ & $-3.55 \pm 4.16$ & \multirow{2}{*}{0.120} & $-3.21 \pm 4.14$ & \multirow{2}{*}{0.471} \\
\hline E only $(\mathrm{n}=53)$ & $3.40 \pm 4.12$ & $1.08 \pm 1.60^{\mathrm{a}}$ & $0.75 \pm 1.51^{\mathrm{a}}$ & $-2.32 \pm 4.04$ & & $-2.64 \pm 4.12$ & \\
\hline \multicolumn{8}{|l|}{ Acid regurgitation } \\
\hline$E+M(n=56)$ & $3.82 \pm 4.68$ & $0.77 \pm 1.35^{\mathrm{a}}$ & $0.55 \pm 1.28^{\mathrm{a}}$ & $-3.05 \pm 4.40$ & \multirow{2}{*}{0.351} & $-3.27 \pm 4.50$ & \multirow{2}{*}{0.235} \\
\hline E only $(\mathrm{n}=53)$ & $3.36 \pm 4.31$ & $1.04 \pm 2.43^{\mathrm{a}}$ & $1.02 \pm 2.59^{\mathrm{a}}$ & $-2.32 \pm 3.71$ & & $-2.34 \pm 3.52$ & \\
\hline \multicolumn{8}{|c|}{ Upper abdominal pain } \\
\hline$E+M(n=56)$ & $2.98 \pm 3.91$ & $0.63 \pm 1.09^{\mathrm{a}}$ & $0.75 \pm 1.71^{\mathrm{a}}$ & $-2.36 \pm 3.95$ & \multirow{2}{*}{0.018} & $-2.23 \pm 4.03$ & \multirow{2}{*}{0.251} \\
\hline E only $(\mathrm{n}=53)$ & $2.42 \pm 3.90$ & $1.70 \pm 3.36$ & $0.96 \pm 2.90^{\mathrm{a}}$ & $-0.72 \pm 3.12$ & & $-1.45 \pm 2.90$ & \\
\hline \multicolumn{8}{|l|}{ Belching } \\
\hline $\mathrm{E}+\mathrm{M}(\mathrm{n}=56)$ & $4.11 \pm 4.88$ & $1.95 \pm 3.29^{\mathrm{a}}$ & $2.04 \pm 3.16^{\mathrm{a}}$ & $-2.16 \pm 3.32$ & \multirow{2}{*}{0.013} & $-2.07 \pm 3.99$ & \multirow{2}{*}{0.258} \\
\hline E only $(\mathrm{n}=53)$ & $3.45 \pm 4.29$ & $3.00 \pm 3.62$ & $2.19 \pm 3.18^{\mathrm{a}}$ & $-0.45 \pm 3.73$ & & $-1.26 \pm 3.37$ & \\
\hline \multicolumn{8}{|l|}{ Dyspepsia } \\
\hline $\mathrm{E}+\mathrm{M}(\mathrm{n}=56)$ & $3.09 \pm 4.97$ & $1.27 \pm 2.78^{\mathrm{a}}$ & $1.09 \pm 2.17^{\mathrm{a}}$ & $-1.82 \pm 4.00$ & \multirow{2}{*}{0.837} & $-2.00 \pm 3.77$ & \multirow{2}{*}{0.981} \\
\hline E only $(\mathrm{n}=53)$ & $3.47 \pm 4.61$ & $1.81 \pm 2.97^{\mathrm{a}}$ & $1.49 \pm 3.10^{\mathrm{a}}$ & $-1.66 \pm 4.16$ & & $-1.98 \pm 4.48$ & \\
\hline \multicolumn{8}{|l|}{ Nausea } \\
\hline $\mathrm{E}+\mathrm{M}(\mathrm{n}=56)$ & $1.30 \pm 2.26$ & $0.84 \pm 2.55$ & $0.64 \pm 2.10$ & $-0.46 \pm 2.97$ & \multirow{2}{*}{0.871} & $-0.66 \pm 2.71$ & \multirow{2}{*}{0.779} \\
\hline E only $(\mathrm{n}=53)$ & $1.42 \pm 2.86$ & $0.87 \pm 2.57$ & $0.89 \pm 2.53$ & $-0.55 \pm 2.29$ & & $-0.53 \pm 2.14$ & \\
\hline
\end{tabular}

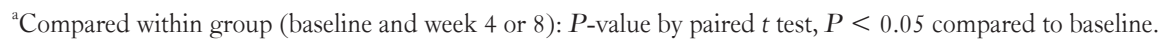

$\mathrm{E}+\mathrm{M}$ group, esomeprazole $40 \mathrm{mg}$ once daily plus mosapride $5 \mathrm{mg} 3$ times daily for 8 weeks; E only group, esomeprazole $40 \mathrm{mg}$ once daily plus placebo 5 mg 3 times daily for 8 weeks. 
line for both treatment groups, but a statistically significant decrease was observed only in the $\mathrm{E}+\mathrm{M}$ group $(\mathrm{E}+\mathrm{M}$ group, $P<0.001$; E only group, $P=0.101)$. When the change in upper abdominal pain scores relative to the baseline was analyzed at 8 weeks, a statistically significant decrease in both groups was observed, similar to the $\mathrm{PP}$ analysis population (E+M group, $P<0.001$; $\mathrm{E}$ only group, $P$ $=0.001)($ Table 4$)$

Changes in belching symptom after 4 and 8 weeks of medication. The change in belching scores relative to the baseline at 4 and 8 weeks was analyzed in both groups, but only the $\mathrm{E}+\mathrm{M}$ group showed a statistically significant decrease (4 weeks: $\mathrm{E}+\mathrm{M}$ group, $P<0.001$; $\mathrm{E}$ only group, $P=0.104 ; 8$ weeks: $\mathrm{E}+\mathrm{M}$ group, $P<0.001 ; \mathrm{E}$ only group, $P=0.001)$. The change in belching scores after 4 weeks showed an almost significant difference between the 2 groups $(P=0.060)$ (Table 3$)$. The ITT analysis population showed similar results to the $\mathrm{PP}$ analysis population, except that the change relative to the baseline at 4 weeks showed a significantly greater improvement in the $\mathrm{E}+\mathrm{M}$ group than in the E only group $(P=0.013)$ (Table 4$)$.

Changes in dyspepsia symptom after 4 and 8 weeks of medication. In the PP analysis population, the change in total dyspepsia scores relative to the baseline after 4 and 8 weeks of medication showed a statistically significant decrease in both treatment groups (4 weeks: $\mathrm{E}+\mathrm{M}$ group, $P=0.008$; E only group, $P$ $=0.004 ; 8$ weeks: $\mathrm{E}+\mathrm{M}$ group, $P=0.002 ; \mathrm{E}$ only group, $P=$ 0.001 ) (Table 3).

Changes in nausea symptom after 4 and 8 weeks of medication. In the $\mathrm{PP}$ analysis population, the change in total nausea scores relative to the baseline at 4 weeks was analyzed for each group, but only the $\mathrm{E}$ only group showed a statistically significant decrease ( $\mathrm{E}+\mathrm{M}$ group, $P=0.097$; E only group, $P=0.019$ ). The change in total nausea scores relative to the baseline at eight weeks showed a significant decrease in both groups ( $\mathrm{E}+\mathrm{M}$ group, $P=0.009$; E only group, $P=0.010$ ) (Table 3 ). In the ITT analysis population, the change relative to the baseline at 8 weeks did not show a significant change in either group $(\mathrm{E}+\mathrm{M}$ group, $P=0.074$; E only group, $P=0.079$ ) (Table 4).

\section{Change in the frequency of individuals showing re- duced upper abdominal pain or dyspepsia after 8 weeks of medication}

The frequency of participants showing a reduction in upper abdominal pain scores relative to the baseline was analyzed for the PP

Table 5. Frequency of Patients with Decreased Upper Abdominal Pain Score Compared to the Baseline

\begin{tabular}{clccc}
\hline \multirow{2}{*}{ Subjects } & Change & E+M group & E only group & P-value \\
\cline { 3 - 4 } & & & Frequency $(\%)$ & \\
PP & Decreased & $29(60.4)$ & $21(48.8)$ & 0.370 \\
$(\mathrm{n}=91)$ & Not decreased & $19(39.6)$ & $22(51.2)$ & 0.155 \\
ITT & Decreased & $34(60.7)$ & $29(45.3)$ & \\
$(\mathrm{n}=109)$ & Not decreased & $22(39.3)$ & $29.7)$ & \\
\hline
\end{tabular}

PP, per-protocol; ITT, intention-to-treat; E+M group, esomeprazole $40 \mathrm{mg}$ once daily plus mosapride $5 \mathrm{mg} 3$ times daily for 8 weeks; E only group, esomeprazole $40 \mathrm{mg}$ once daily plus placebo $5 \mathrm{mg} 3$ times daily for 8 weeks.

Table 6. Frequency of Patients with Decreased Dyspepsia Score Compared to the Baseline

\begin{tabular}{|c|c|c|c|c|}
\hline \multirow{2}{*}{ Subjects } & \multirow{2}{*}{ Change } & $\mathrm{E}+\mathrm{M}$ group & E only group & \multirow{2}{*}{$P$-value } \\
\hline & & \multicolumn{2}{|c|}{ Frequency (\%) } & \\
\hline $\mathrm{PP}$ & Decreased & $25(52.1)$ & $21(48.8)$ & 0921 \\
\hline$(\mathrm{n}=91)$ & Not decreased & $23(47.9)$ & $22(51.2)$ & 0.921 \\
\hline ITT & Decreased & $30(53.6)$ & $25(47.2)$ & 0.634 \\
\hline$(\mathrm{n}=109)$ & Not decreased & $26(46.4)$ & $28(52.8)$ & \\
\hline
\end{tabular}

PP, per-protocol; ITT, intention-to-treat; E+M group, esomeprazole $40 \mathrm{mg}$ once daily plus mosapride $5 \mathrm{mg} 3$ times daily for 8 weeks; $\mathrm{E}$ only group, esomeprazole $40 \mathrm{mg}$ once daily plus placebo $5 \mathrm{mg} 3$ times daily for 8 weeks. 
and ITT analysis populations. There was no statistically significant difference in frequency between the 2 treatment groups for either analysis population (PP, $P=0.370$; ITT, $P=0.155$ ) (Table 5). The frequency of participants displaying a reduction in dyspepsia scores was also analyzed, and this also did not show a statistically significant difference between the 2 treatment groups for either analysis population ( $\mathrm{PP}, P=0.921$; ITT, $P=0.634$ ) (Table 6).

\section{Improved rates in total gastroesophageal reflux disease symptoms after 4 and 8 weeks of medication}

In ITT analysis population, the proportion of patients showing a reduction in total GERD scores after 4 weeks more than $25 \%$ and $50 \%$ was significantly higher in the $\mathrm{E}+\mathrm{M}$ group than the $\mathrm{E}$ only group ( $P=0.038$ and $P=0.028$, respectively) (Fig. 2).

\section{Safety Assessment}

The number of participants showing an adverse event during the 8 weeks of treatment was $12(20.3 \%)$ in the $\mathrm{E}+\mathrm{M}$ group and $11(20.4 \%)$ in the E only group, which was not a statistically significant difference between the 2 treatment groups $(P=0.997)$. Measurements from laboratory tests were defined as normal or abnormal based on reference values. None of the measured values showed a significant change in the frequency of normal values after treatment. In the $\mathrm{E}+\mathrm{M}$ group, diastolic blood pressure (DBP) showed a significant decrease after four weeks of medication $(P$ $=0.043)$, while systolic blood pressure (SBP) and DBP both decreased significantly relative to the baseline after 8 weeks (SBP, $P=0.027$; DBP, $P=0.003$ ). In the $\mathrm{E}$ only group, body weight increased significantly after 8 weeks of medication $(P=0.012)$. None of the other variables analyzed showed a significant difference relative to the baseline at any of the time point analyzed.

\section{Discussion}

This study aimed to evaluate the safety and efficacy of a combination treatment with esomeprazole and mosapride $(\mathrm{E}+\mathrm{M})$ in patients with ERD, by comparing the endoscopic healing rate according to the LA classification obtained with the combination treatment with esomeprazole only (E only) treatment. As shown in the results above, the primary outcome (endoscopic amelioration) showed a significant improvement in both groups, and there was no difference between the $\mathrm{E}+\mathrm{M}$ and $\mathrm{E}$ only groups. In the ITT analysis population, among the results for secondary outcomes (GERD symptoms), upper abdominal pain, belching, and total GERD score showed a significantly greater improvement relative to the baseline in the $\mathrm{E}+\mathrm{M}$ group than in the $\mathrm{E}$ only group after 4 weeks of treatment. Mosapride has a strong effect on belching; hence, although there was some improvement in the symptom with esomeprazole alone, the symptom improved more rapidly when esomeprazole was combined with mosapride. In the analysis of GERD total scores, the ratio of patients with an improvement in symptoms of at least $25 \%$ or at least $50 \%$ at 4 weeks after medication was significantly higher in the $\mathrm{E}+\mathrm{M}$ group, which demonstrates that administration of esomeprazole and mosapride together has a more rapid initial symptom improvement in some specific GERD symptoms, such as upper abdominal pain and belching.

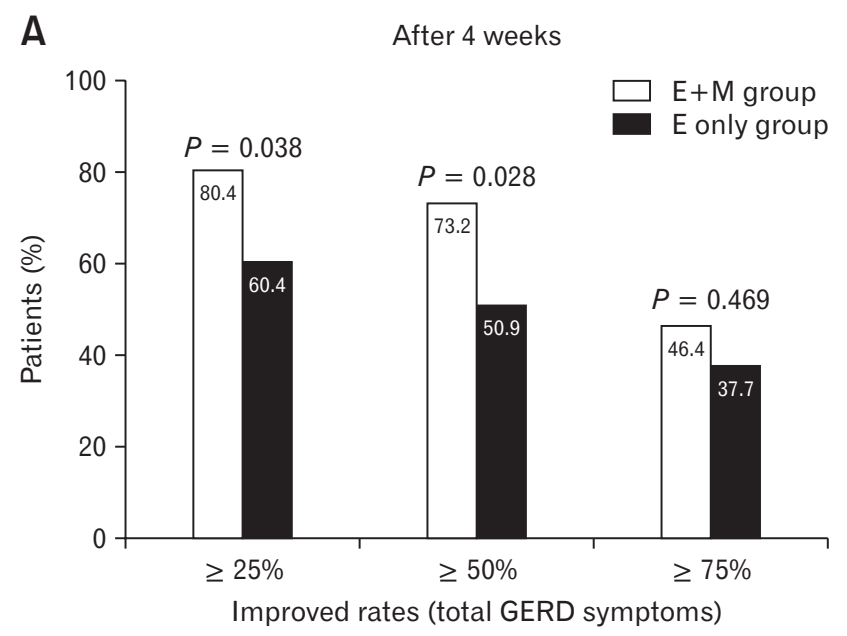

B

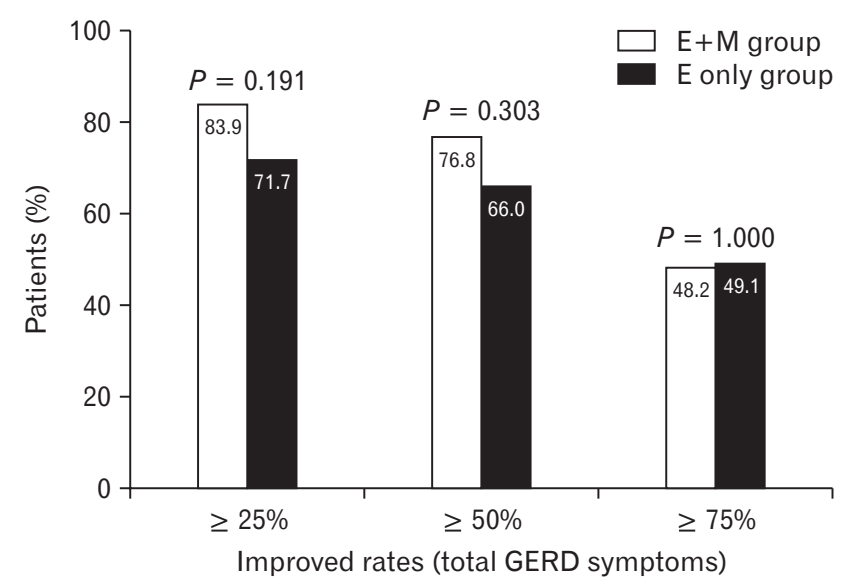

Figure 2. Improved rates in total gastroesophageal reflux disease symptoms after 4 and 8 weeks of medication (intention-to-treat analysis). E $+\mathrm{M}$ group, esomeprazole $40 \mathrm{mg}$ once daily plus mosapride $5 \mathrm{mg} 3$ times daily for 8 weeks; E only group, esomeprazole 40 mg once daily plus placebo $5 \mathrm{mg} 3$ times daily for 8 weeks. 
Since PPIs are unstable at low $\mathrm{pH}$, a long gastric retention time as the result of dysmotility can impair the effect of PPI in inhibiting gastric acid. ${ }^{14,15}$ Therefore, it is beneficial for PPIs to move rapidly into the upper small intestine. In other clinical studies, when a PPI (rabeprazole) was administered in combination with a prokinetic drug, the prokinetic enabled faster transit of the PPI from the stomach to the small intestine, which means that the higher PPI levels were in a stable state in the small intestine, significantly increasing the blood PPI concentration, and suggesting that prokinetics promote PPIs absorption. ${ }^{16}$ Given this result, the prokinetic mosapride in the present study may also be responsible for the increased absorbance of the PPI esomeprazole, thereby enabling a more rapid initial improvement in the $\mathrm{E}+\mathrm{M}$ group.

Madan et $\mathrm{al}^{11}$ compared the effects of pantoprazole and mosapride combination therapy with pantoprazole alone in ERD and NERD patients, and found that GERD symptoms scores were significantly lower in the combination therapy group after 8 weeks of treatment. In particular, they reported that although there was a significant improvement in symptoms with the combination therapy for ERD, no difference was reported between the 2 groups in endoscopic healing.

In a randomized, controlled trial, Hsu et al ${ }^{17}$ performed 4 weeks of lansoprazole and mosapride combination or lansoprazole alone therapies in ERD patients who had been diagnosed endoscopically. However, there was no difference in GERD symptoms between the 2 groups after treatment (combination therapy 13.4 vs single treatment $10.9, P=0.103$ ); based on a subgroup analysis, the authors reported that although combination therapy with mosapride had only a small effect on ERD patients, it could still be beneficial for patients with severe symptoms. Another study from Japan reported similar results: although there was no significant improvement in the overall symptoms scores in a combination therapy group, belching symptoms did improve significantly in the single treatment group, and that combination therapy was more effective in ERD than NERD patients. ${ }^{18}$ A study from Korea reported that a gastric emptying scan showed an effect for mosapride combination therapy in preventing delayed gastric emptying, and that postprandial bloating and nausea symptoms improved more in the combination therapy group than in the single treatment group. ${ }^{19}$ Cho et $\mathrm{al}^{20}$ compared esomeprazole and mosapride combination therapy with esomeprazole alone treatment and they found that although there was no difference in the treatment response between the 2 groups, an assessment of esophageal peristaltic function using high-resolution manometry showed that there was a greater decrease in intrabolus pressure and an increase in esophageal contract- ibility relative to the baseline in the combination therapy group than in the single treatment group. Therefore, addition of prokinetics is thought to be useful in GERD patients due to the aforementioned mechanisms.

Moreover, in terms of dyspepsia and acid regurgitation symptoms, the fact that improvements at 8 weeks of medication were greater than those at 4 weeks demonstrates that long-term treatment is required to ease these symptoms. Nausea showed a lower baseline score than other symptoms, resulting in a difficult evaluation of the difference in improvement between the 2 groups.

This study is a double-blind, randomized clinical trial which minimized the bias and confounders. Moreover, we excluded NERD patients and enrolled only symptomatic ERD patients which reduced the heterogeneity of patients. However, our results did not show definite differences between the 2 groups in most of the GERD sub-symptoms that possibly originated from small sample size. Thus, future studies with larger sample sizes will be needed. Another strength of this study is the 8-week treatment period and that evaluated the long term effect of combination therapy. However, the GERD symptoms score questionnaire that was used in this study has some limitations. In fact, it is difficult to distinguish belching from dyspepsia or nausea. Furthermore, we cannot clearly rule out overlap syndrome that overlaps GERD symptoms and dyspepsia. In this study, the endoscopic healing rate at 8 weeks of treatment is lower (60.5\% in $\mathrm{E}$ only group) than that in a conventional study which report a $92.6 \%$ healing rate in an esomeprazole alone group. ${ }^{21}$ However, the conventional study analyzed the healing rate by different methods using cumulative life-table healing rates that compensates for the actual time of healing, ${ }^{21}$ and the baseline LA classification of patients are also different from the present study.

In conclusion, ERD patients did not show a statistically significant difference in the endoscopic healing rate according to the LA classification when given esomeprazole in combination with mosapride or esomeprazole alone. However, the combination therapy showed a tendency for upper abdominal pain, belching, and total GERD symptoms scores to improve more rapidly. This suggests that combination therapy with esomeprazole and mosapride will be useful for rapid improvement of specific GERD symptoms, such as upper abdominal pain and belching in ERD patients.

Financial support: This study was sponsored by Hanmi Pharmaceutical Co, Ltd, Seoul, Korea.

Conflicts of interest: None. 
Author contributions: Ju Yup Lee analyzed and interpreted the data and wrote a manuscript; Sung Kook Kim designed, organized, and mediated the present study, and supervised the manuscript; Kwang Bum Cho, Joong Goo Kwon, and Byung Ik Jang supervised study design and the manuscript; and Kyung Sik Park, Jin Tae Jung, Eun Young Kim, and Si Hyung Lee enrolled the study patients.

\section{References}

1. Vakil N, van Zanten SV, Kahrilas P, Dent J, Jones R. The Montreal definition and classification of gastroesophageal reflux disease: a global evidence-based consensus. Am J Gastroenterol 2006;101:1900-1920.

2. Fock KM, Talley NJ, Fass R, et al. Asia-Pacific consensus on the management of gastroesophageal reflux disease: update. J Gastroenterol Hepatol 2008;23:8-22.

3. Min YW, Shin YW, Cheon GJ, et al. Recurrence and its impact on the health-related quality of life in patients with gastroesophageal reflux disease: a prospective follow-up analysis. J Neurogastroenterol Motil 2016;22:86-93.

4. Kim SE, Kim N, Oh S, et al. Predictive factors of response to proton pump inhibitors in Korean patients with gastroesophageal reflux disease. J Neurogastroenterol Motil 2015;21:69-77.

5. Goh KL, Choi MG, Hsu PI, et al. Pharmacological and safety profile of dexlansoprazole: a new proton pump inhibitor - implications for treatment of gastroesophageal reflux disease in the Asia pacific region. J Neurogastroenterol Motil 2016;22:355-366.

6. Jung HK, Hong SJ, Jo YJ, et al. [Updated guidelines 2012 for gastroesophageal reflux disease]. Korean J Gastroenterol 2012;60:195-218. [Korean]

7. Katz PO, Gerson LB, Vela MF. Guidelines for the diagnosis and management of gastroesophageal reflux disease. Am J Gastroenterol 2013;108:308-328.

8. Boeckxstaens GE. Review article: the pathophysiology of gastro-oesophageal reflux disease. Aliment Pharmacol Ther 2007;26:149-160.

9. Fujiwara Y, Takahashi S, Arakawa T, et al. A 2008 questionnaire-based survey of gastroesophageal reflux disease and related diseases by physicians in East Asian countries. Digestion 2009;80:119-128.
10. Miwa H, Inoue K, Ashida K, et al. Randomised clinical trial: efficacy of the addition of a prokinetic, mosapride citrate, to omeprazole in the treatment of patients with non-erosive reflux disease - a double-blind, placebo-controlled study. Aliment Pharmacol Ther 2011;33:323-332.

11. Madan K, Ahuja V, Kashyap PC, Sharma MP. Comparison of efficacy of pantoprazole alone versus pantoprazole plus mosapride in therapy of gastroesophageal reflux disease: a randomized trial. Dis Esophagus 2004;17:274-278.

12. Ren LH, Chen WX, Qian LJ, Li S, Gu M, Shi RH. Addition of prokinetics to PPI therapy in gastroesophageal reflux disease: a meta-analysis. World J Gastroenterol 2014;20:2412-2419.

13. Nayar DS, Vaezi MF. Classifications of esophagitis: who needs them? Gastrointest Endosc 2004;60:253-257.

14. Ndraha S. Combination of PPI with a prokinetic drug in gastroesophageal reflux disease. Acta Med Indones 2011;43:233-236.

15. Miyamoto M, Haruma K, Takeuchi K, Kuwabara M. Frequency scale for symptoms of gastroesophageal reflux disease predicts the need for addition of prokinetics to proton pump inhibitor therapy. J Gastroenterol Hepatol 2008;23:746-751.

16. Arai K, Takeuchi Y, Watanabe H, Tsukurimichi A, Uchida N, Imawari M. Prokinetics influence the pharmacokinetics of rabeprazole. Digestion 2008;78:67-71.

17. Hsu YC, Yang TH, Hsu WL, et al. Mosapride as an adjunct to lansoprazole for symptom relief of reflux oesophagitis. Br J Clin Pharmacol 2010;70:171-179.

18. Yamaji Y, Isomura Y, Yoshida S, Yamada A, Hirata Y, Koike K. Randomized controlled trial comparing the efficacy of mosapride plus omeprazole combination therapy to omeprazole monotherapy in gastroesophageal reflux disease. J Dig Dis 2014;15:469-476.

19. Lim HC, Kim JH, Youn YH, Lee EH, Lee BK, Park H. Effects of the addition of mosapride to gastroesophageal reflux disease patients on proton pump inhibitor: a prospective randomized, double-blind study. J Neurogastroenterol Motil 2013;19:495-502.

20. Cho YK, Choi MG, Park EY, et al. Effect of mosapride combined with esomeprazole improves esophageal peristaltic function in patients with gastroesophageal reflux disease: a study using high resolution manometry. Dig Dis Sci 2013;58:1035-1041.

21. Castell DO, Kahrilas PJ, Richter JE, et al. Esomeprazole (40 mg) compared with lansoprazole $(30 \mathrm{mg})$ in the treatment of erosive esophagitis. Am J Gastroenterol 2002;97:575-583. 